\title{
Generalized su $(1,1)$ coherent states for pseudo harmonic oscillator and their nonclassical properties
}

\author{
B. Mojaveri* and A. Dehghani ${ }^{\dagger}$ \\ Department of Physics, Azarbaijan Shahid Madani University, PO Box 51745-406, Tabriz, Iran \\ Department of Physics, Payame Noor University, PO Box 19395-4697, Tehran, Iran
}

March 20, 2018

\begin{abstract}
In this paper we define a non-unitary displacement operator, which by acting on the vacuum state of the pseudo harmonic oscillator ( $\mathrm{PHO}$ ), generates new class of generalized coherent states (GCSs). An interesting feature of this approach is that, contrary to the Klauder-Perelomov and Barut-Girardello approaches, it does not require the existence of dynamical symmetries associated with the system under consideration. These states admit a resolution of the identity through positive definite measures on the complex plane. We have shown that the realization of these states for different values of the deformation parameters leads to the well-known Klauder-Perelomov and Barut-Girardello CSs associated with the $s u(1,1)$ Lie algebra. This is why we call them the generalized $s u(1,1)$ CSs for the PHO. Finally, study of some statistical characters such as squeezing, anti-bunching effect and sub-Poissonian statistics reveals that the constructed GCSs have indeed nonclassical features.
\end{abstract}

Keywords: Nonlinear Coherent States, Pseudo Harmonic Oscillator, Sub-Poissonian Statistics, Squeezing Effect.

\section{Introduction}

Coherent states (CSs), were first established by Schrödinger [1] as the eigenvectors of the boson annihilation operator, $\hat{a}$, corresponding to the Heisenbereg-Weyl Lie algebra. They play an important role in quantum optics and provide us with a link between quantum and classical oscillators. Moreover, these states can be produced by acting of the Glauber displacement operator, $D(z)=e^{z \hat{a}^{\dagger}-\bar{z} \hat{a}}$, on the vacuum states, where $z$ is a complex variable. These states were later applied successfully to some other models based on their Lie algebra symmetries by Glauber [2, 3], Klauder [4, 5], Sudarshan [6], Barut and Girardello [7] and Perelomov [8]. Additionally, for the models with one degree of freedom either discrete or

\footnotetext{
*Email: bmojaveri@azaruniv.ac.ir; bmojaveri@gmail.com

†Email: a_dehghani@tabrizu.ac.ir, alireza.dehghani@gmail.com
} 
continuous spectra- with no remark on the existence of a Lie algebra symmetry- Gazeau et al proposed new CSs, which were parametrized by two real parameters [9, 10]. Moreover, there exist some considerations in connection with CSs corresponding to the shape invariance symmetries [11, 12]. To construct CSs, four main different approaches the so-called Schrödinger, Klauder-Perelomov, Barut-Girardello, and Gazeau-Klauder methods have been found, so that the second and the third approaches rely directly on the Lie algebra symmetries and their corresponding generators. Here, it is necessary to emphasize that quantum coherence of states nowadays pervade many branches of physics such as quantum electrodynamics, solid-state physics, and nuclear and atomic physics, from both theoretical and experimental viewpoints.

In addition to CSs, squeezed states (SSs) are becoming increasingly important. These are the non-classical states of the electromagnetic field in which certain observables exhibit fluctuations less than in the vacuum state [13]. These states are important because they can achieve lower quantum noise than the zero-point fluctuations of the vacuum or coherent states. Over the last four decades there have been several experimental demonstrations of nonclassical effects, such as the photon anti-bunching [14, sub-Poissonian statistics [15, 16], and squeezing [17, 18. On the other hand, considerable attention has been paid to the deformation of the harmonic oscillator algebra of creation and annihilation operators [19]. Some important physical concepts such as the CSs, the even- and odd-CSs for ordinary harmonic oscillator have been extended to deformation case. Moreover, there exist interesting quantum effects, and related quantum states that are namely superposition states exhibiting quantum interference effects [20, 21]. Besides, superpositions of CSs can be prepared in the motion of a trapped ion [22, 23]. With respect to the nonclassical effects, the coherent states turn out to define the limit between the classical and nonclassical behavior.

Another type of generalization of CSs is the nonlinear coherent states (NLCSs), or fCSs. The NLCSs, $|z, f\rangle$, are right-hand eigenstates of the product of nonlinear function $f(\hat{N})$ of the number operator $\hat{N}$ and the boson annihilation operator $\hat{a}$, i.e. they satisfy $f(\hat{N}) \hat{a}|z, f\rangle=z|z, f\rangle$. The nature of the nonlinearity depends on the choice of the function $f(\hat{N})$ [24]. These states may appear as stationary states of the center-of mass motion of a trapped ion [25, 26]. NLCSs exhibit nonclassical features such as quadrature squeezing, sub-Poissonian statistics, anti-bunching, self-splitting effects and so on [27-34].

The $s u(1,1)$ Lie algebra is of great interest in quantum optics because it can characterize many kinds of quantum optical systems [7, 8, 35, 36]. It has recently been used by many researchers to investigate the nonclassical properties of light in quantum optical systems [37]. In particular, the bosonic realization of $s u(1,1)$ describes the degenerate and non-degenerate parametric amplifiers [38]. The squeezed states and nonlinear SSs of photons have been considered in terms of $s u(1,1)$ Lie algebra and the CSs associated with this algebra [39].

In the present paper, we want to construct new type NLCSs for pseudo harmonic oscillator (PHO). This exactly solvable quantum model is the sum of the harmonic oscillator and the inversely quadratic potential was proposed by Goldman and Krivchenkov [40], i.e.

$$
V(x)=\left[\frac{1}{2} \mu w^{2} x^{2}+\frac{\hbar}{2 \mu} \frac{\lambda(\lambda-1)}{x^{2}}\right],
$$

where $m, w$ and $\lambda$ respectively represent the mass of the particle, the frequency and the strength of the external field. Sometimes this system has been called isotonic potential 
[41, 42, 43]. PHO may be more suitable model for the description of vibrating molecules. For this reason, the study of CSs for PHO is of great importance which has recently been studied Klauder-Perelomov and Barut-Girardello type CSs in the framework of $s u(1,1)$ Lie algebra symmetry [44, 45, 46].

The aim of this work is introducing a new approach to construct GCSs for PHO. This approach based on the generalization of the displaced operator associated with su(1,1) Lie algebra which will be acting on the vacuum stats of PHO. This approach is extension of the our previous work in connection to new type NLCSs for harmonic oscillator associated with the Heisenbereg-Weyl algebra [47. An interesting feature of this approach is that, Contrary to the Klauder-Perelomov and Barut-Girardello approach, this does not require for existence of dynamical symmetries associated with the considered system. To construction of such states, we need only to the raising operator associated with the considered system in the framework of supersymmetric quantum mechanics. These states admit a resolution of the identity through positive definite and non-oscillating measures on the complex plane. We have shown that these states are NLCSs and for different values of the deformation parameter lead to the well-known Klauder-Perelomov and Barut-Girardello CSs for PHO. Some interesting features are found. For instance, we have shown that they evolve in time as like as the canonical CSs, in other words the constructed GCSs possess the temporal stability property. Furthermore, it has been discussed in detail that they have indeed nonclassical features such as squeezing, anti-bunching effect and sub-Poissonian statistics, too.

This paper is organized as follows: in section 2, we briefly review on a su(1,1) Lie algebra symmetry of PHO and construct the new CSs $|z\rangle_{r}^{\lambda}$, via generalized analogue of the displacement operators acting on the vacuum state. In order to realize the resolution of the identity, we have found the positive definite measures on the complex plane. With a review on these states, the relation between su $(1,1)$ Klauder-Perelomov and Barut-Girardello CSs of PHO with constructed CSs will be obvious. It has been shown that these states can be considered as eigenstates of a certain annihilation operator, then they can be interpreted as NLCSs with a special nonlinearity function. Furthermore, in section 3 by evaluating some physical quantities, we discuss their non-classical properties. Finally, we conclude the paper in section 4 .

\section{New GCSs for PHO}

In Refs. [45, 48, 49], it has been shown that the second-order differential operators

$$
\begin{aligned}
& J_{ \pm}^{\lambda}:=\frac{1}{4}\left[\left(x \mp \frac{d}{d x}\right)^{2}-\frac{\lambda(\lambda-1)}{x^{2}}\right], \\
& J_{3}^{\lambda}:=\frac{H^{\lambda}}{2}=\frac{1}{4}\left[-\frac{d^{2}}{d x^{2}}+x^{2}+\frac{\lambda(\lambda-1)}{x^{2}}\right],
\end{aligned}
$$

satisfy the standard commutation relations of $s u(1,1)$ Lie algebra as follows

$$
\left[J_{+}^{\lambda}, J_{-}^{\lambda}\right]=-2 J_{3}^{\lambda}, \quad\left[J_{3}^{\lambda}, J_{ \pm}^{\lambda}\right]= \pm J_{ \pm}^{\lambda} .
$$

Here, $H^{\lambda}$ is the PHO or Calegero-Sutherland Hamiltonian on the half-line $x$, which satisfy the eigenvalue equation $H^{\lambda}|n, \lambda\rangle=\left(2 n+\lambda+\frac{1}{2}\right)$ for $\mu=\hbar=w=1$. In terms of the Fock 
states, defined by the associated Laguerre polynomials [50] $L_{n}^{\alpha}(x)=\frac{1}{n !} x^{-\alpha} e^{x}\left(\frac{d}{d x}\right)^{n}\left(x^{n+\alpha} e^{-x}\right)$ with $\operatorname{Re}(\alpha)>-1$,

$$
\langle x \mid n, \lambda\rangle=(-1)^{n} \sqrt{\frac{2 \Gamma(n+1)}{\Gamma\left(n+\lambda+\frac{1}{2}\right)}} x^{\lambda} e^{-\frac{x^{2}}{2}} L_{n}^{\lambda-\frac{1}{2}}\left(x^{2}\right), \quad \lambda>\frac{-1}{2},
$$

one can realizes that the infinite dimensional Hilbert space $\mathcal{H}^{\lambda}:=\left.\operatorname{span}\{|n, \lambda\rangle\}\right|_{n=0} ^{\infty}$ products the unitary and positive-integer irreps of $s u(1,1)$ Lie algebra as

$$
\begin{aligned}
& J_{+}^{\lambda}|n-1, \lambda\rangle=\sqrt{n\left(n+\lambda-\frac{1}{2}\right)}|n, \lambda\rangle, \\
& J_{-}^{\lambda}|n, \lambda\rangle=\sqrt{n\left(n+\lambda-\frac{1}{2}\right)}|n-1, \lambda\rangle, \\
& J_{3}^{\lambda}|n, \lambda\rangle=\left(n+\frac{\lambda}{2}+\frac{1}{4}\right)|n, \lambda\rangle .
\end{aligned}
$$

It is straightforward that the orthogonality condition of the associated Laguerre polynomials lead to the following orthogonality condition of the basis of the Hilbert space $\mathcal{H}^{\lambda}$ :

$$
\langle n, \lambda \mid m, \lambda\rangle:=\frac{2 n !}{\Gamma\left(n+\lambda+\frac{1}{2}\right)} \int_{0}^{\infty} x^{2 \lambda} e^{-x^{2}} L_{n}^{\lambda-\frac{1}{2}}\left(x^{2}\right) L_{m}^{\lambda-\frac{1}{2}}\left(x^{2}\right) d x=\delta_{n m} .
$$

It is useful to stress that the two operators $J_{+}^{\lambda}$ and $J_{-}^{\lambda}$ are Hermitian conjugate of each others with respect to the inner product (5) and $J_{3}^{\lambda}$ is self-adjoint operator, too.

According to the definition has already been given in Ref. [47], the following GCSs for PHO are produced, here, via generalized analogue of the displacement operators acting on the vacuum state of $\mathrm{PHO},|0, \lambda\rangle$

$$
|z\rangle_{r}^{\lambda}:=M_{r}^{\lambda^{-\frac{1}{2}}}(|z|)_{1} F_{r}\left(\left[\lambda-\frac{1}{2}\right],\left[\lambda-\frac{1}{2}, \lambda+\frac{1}{2}, \ldots, \lambda-\frac{3}{2}+r\right], z J_{+}^{\lambda}\right)|0, \lambda\rangle, \quad r \geq 1
$$

where ${ }_{p} F_{q}(\ldots)$ is the generalized hypergeometric function and $z\left(=|z| e^{i \varphi}\right)$ and $r$ are respectively the coherence and the deformation parameters, respectively. Now, we show that the well known su(1,1) Klauder-Perelomov and Barut-Girardello CSs can be considered as a special case of introduced GCSs. Clearly, $|z\rangle_{r}^{\lambda}$ becomes the $s u(1,1)$ Klauder-Perelomov CSs for the PHO, $|z\rangle_{K P}^{\lambda}$ [45, 46]), when $r$ tends to unity and $z$ be replaced with $\frac{z}{|z|} \tanh (|z|)$. Using the series form of the hypergeometric functions and applying the laddering relations, Eqs. (5), $|z\rangle_{r}^{\lambda}$ can be expanded into the basis $|n, k\rangle$ as

$$
|z\rangle_{r}^{\lambda}=M_{r}^{\lambda^{-\frac{1}{2}}}(|z|) \sum_{n=0}^{\infty} z^{n} \prod_{k=1}^{r-1} \frac{\Gamma\left(\lambda+k-\frac{1}{2}\right)}{\Gamma\left(n+\lambda+k-\frac{1}{2}\right)} \sqrt{\frac{\Gamma\left(n+\lambda+\frac{1}{2}\right)}{\Gamma\left(\lambda+\frac{1}{2}\right) \Gamma(n+1)}}|n, \lambda\rangle, \quad r \geq 2,
$$

where $M_{r}^{\lambda}(|z|)$ is chosen so that $|z\rangle_{r}^{\lambda}$ is normalized, i.e. ${ }_{r}^{\lambda}\langle z \mid z\rangle_{r}^{\lambda}=1$, then

$$
M_{r}^{\lambda}(|z|)={ }_{1} F_{2 r-2}\left(\left[\lambda+\frac{1}{2}\right],\left[\lambda+\frac{1}{2}, \lambda+\frac{1}{2}, \ldots, \lambda-\frac{3}{2}+r, \lambda-\frac{3}{2}+r\right],|z|^{2}\right) .
$$


Now, one can check that for the case $r=2$, we have

$$
\begin{aligned}
|z\rangle_{2}^{\lambda} & =M_{2}^{\lambda^{-\frac{1}{2}}}(|z|)_{1} F_{2}\left(\left[\lambda-\frac{1}{2}\right],\left[\lambda-\frac{1}{2}, \lambda+\frac{1}{2}\right], z J_{+}^{\lambda}\right)|0, \lambda\rangle \\
& =M_{2}^{\lambda^{-\frac{1}{2}}}(|z|) \sum_{n=0}^{\infty} z^{n} \sqrt{\frac{\Gamma\left(\lambda+\frac{1}{2}\right)}{\Gamma(n+1) \Gamma\left(n+\lambda+\frac{1}{2}\right)}}|n, \lambda\rangle,
\end{aligned}
$$

meanwhile it satisfies following eigenvalue equation

$$
J_{-}^{\lambda}|z\rangle_{2}^{\lambda}=z|z\rangle_{2}^{\lambda}
$$

Then, $|z\rangle_{r \rightarrow 2}^{\lambda}$ is reduced to the $s u(1,1)$ coherency of the Barut-Girardello type, has already given in Ref [45, 46] corresponding to the PHO model. Also, it should be noticed that, these states can be categorized as special class of Generalized Hypergeometric CSs [51] have already been made by Appl et al.

Using the inner product (6), the overlapping of the GCSs can be calculated as follows:

$$
\begin{aligned}
& { }_{r}^{\lambda}\left\langle z_{1} \mid z_{2}\right\rangle_{r}^{\lambda}=\frac{{ }_{1} F_{2 r-2}\left(\left[\lambda+\frac{1}{2}\right],\left[\lambda+\frac{1}{2}, \lambda+\frac{1}{2}, \ldots, \lambda-\frac{3}{2}+r, \lambda-\frac{3}{2}+r\right], \bar{z}_{1} z_{2}\right)}{\sqrt{M_{r}^{\lambda}\left(\left|z_{1}\right|\right) M_{r}^{\lambda}\left(\left|z_{2}\right|\right)}}, \\
& { }_{r_{1}}^{\lambda}\langle z \mid z\rangle_{r_{2}}^{\lambda}=\frac{{ }_{1} F_{r_{1}+r_{2}-2}\left(\left[\lambda+\frac{1}{2}\right],\left[\lambda+\frac{1}{2}, \lambda+\frac{1}{2}, \ldots, \lambda-\frac{3}{2}+r_{1}, \lambda-\frac{3}{2}+r_{2}\right],|z|^{2}\right)}{\sqrt{M_{r_{1}}^{\lambda}(|z|) M_{r_{2}}^{\lambda}(|z|)}},
\end{aligned}
$$

and result that two different kinds of these states are non-orthogonal, if $r_{1} \neq r_{2}, z_{1} \neq z_{2}$. Now, we are in a position to introduce the appropriate measure $d \mu_{r}(|z|):=K_{r}^{\lambda}(|z|) \frac{d|z|^{2} d \varphi}{2}$ so that the resolution of the identity is realized for the GCSs $|z\rangle_{r}^{\lambda}$ in the Hilbert space $\mathcal{H}^{\lambda}$ :

$$
\begin{aligned}
& 1_{\mathcal{H}^{\lambda}}=\oint_{\mathbb{C}(z)}|z\rangle_{r}^{\lambda} \underset{r}{\lambda}\langle z| d \mu_{r}(|z|) \\
& =2 \pi \sum_{n=0}^{\infty}\left[\prod_{k=1}^{r-1} \frac{\Gamma\left(\lambda+k-\frac{1}{2}\right)}{\Gamma\left(n+\lambda+k-\frac{1}{2}\right)}\right]^{2} \frac{\Gamma\left(n+\lambda+\frac{1}{2}\right)}{\Gamma\left(\lambda+\frac{1}{2}\right) \Gamma(n+1)}|n, \lambda\rangle\left\langle n,\left.\lambda\left|\int_{0}^{\infty}\right| z\right|^{2 n+1} \frac{K_{r}^{\lambda}(|z|)}{M_{r}^{\lambda}(|z|)} d|z| .\right.
\end{aligned}
$$

It is found that using by the integral relation for the Meijers G-functions (see $\frac{7-811}{4}$ in [50]), these states resolve the unity operator for any $r$ and $\lambda$ through a positive definite and nonoscillating measure

$$
\begin{aligned}
K_{r}^{\lambda}(|z|)= & \frac{\Gamma\left(\lambda+\frac{1}{2}\right)_{1} F_{2 r-2}\left(\left[\lambda+\frac{1}{2}\right],\left[\lambda+\frac{1}{2},, \lambda+\frac{1}{2}, \ldots, \lambda-\frac{3}{2}+r, \lambda-\frac{3}{2}+r\right],|z|^{2}\right)}{\pi\left[\prod_{k=1}^{r-1} \Gamma\left(\lambda+k-\frac{1}{2}\right)\right]^{2}} \\
& \times G_{2,2 r}^{2 r-1,1}\left(\left.|z|^{2}\right|_{0, \lambda-\frac{1}{2}, \lambda-\frac{1}{2}, \ldots, \lambda+r-\frac{5}{2}, \lambda+r-\frac{5}{2}, 0} ^{0, \lambda-\frac{1}{2}}\right) .
\end{aligned}
$$

For $(\lambda ; r)=\left(\frac{3}{4} ; 1\right)$ as well as $(\lambda ; r)=\left(\frac{3}{2} ; 2,3\right.$ and 4$)$ we have plotted the changes of the non-oscillating and positive definite measures $K_{r}^{\lambda}(|z|)$ in terms of $|z|^{2}$ in Figures 1 (a) and 1(b), respectively. 

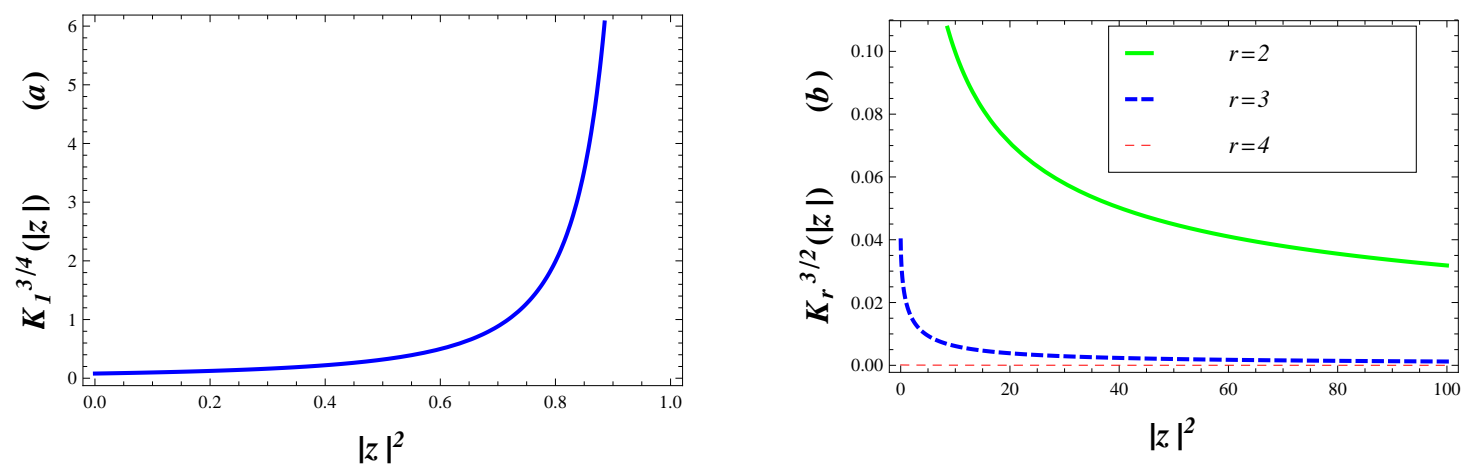

Figure 1:

- Figure. 1. Plots of the positive definite measures $K_{r}^{\lambda}(|z|)$ in terms of $|z|^{2}$; for $\lambda=\frac{3}{4}$ and $r=1$ in (a) likewise $\lambda=\frac{3}{2}$ and $r(=2,3$ and 4$)$ in $(b)$.

$\diamond$ Coordinate Representation of $|z\rangle_{r}^{\lambda}$

Based on a new expression of the Laguerre polynomials as an operator-valued function given in $[52]$

$$
L_{n}^{\alpha}(y)=\frac{1}{n !} y^{-\alpha}\left(\frac{d}{d y}-1\right)^{n} y^{n+\alpha},
$$

also, according to Eqs. (4) and (8) we have

$$
\begin{aligned}
& \langle x \mid z\rangle_{r}^{\lambda}=\sqrt{\frac{2}{M_{r}^{\lambda}(|z|) \Gamma\left(\lambda+\frac{1}{2}\right)}} \sum_{n=0}^{\infty}(-z)^{n} \prod_{k=1}^{r-1} \frac{\Gamma\left(\lambda+k-\frac{1}{2}\right)}{\Gamma\left(n+\lambda+k-\frac{1}{2}\right)} x^{\lambda} e^{-\frac{x^{2}}{2}} L_{n}^{\lambda-\frac{1}{2}}\left(x^{2}\right) \\
& =\sqrt{\left.\frac{2}{M_{r}^{\lambda}(|z|) \Gamma\left(\lambda+\frac{1}{2}\right)} \sum_{n=0}^{\infty} \frac{(-z)^{n}}{n !} \prod_{k=1}^{r-1} \frac{\Gamma\left(\lambda+k-\frac{1}{2}\right)}{\Gamma\left(n+\lambda+k-\frac{1}{2}\right)} e^{-\frac{y}{2}} y^{\frac{1-\lambda}{2}}\left(\frac{d}{d y}-1\right)^{n} y^{n+\lambda-\frac{1}{2}}\right|_{y=x^{2}} .}
\end{aligned}
$$

Along with substitution

$$
\left(\frac{d}{d y}-1\right)^{n} y^{n}=\left(y \frac{d}{d y}+n-y\right) \ldots\left(y \frac{d}{d y}+1-y\right)=\left(y \frac{d}{d y}-y+1\right)_{n},
$$

it becomes

$$
\begin{aligned}
& \langle x \mid z\rangle_{r}^{\lambda}=\left.\left[\frac{2 e^{-y} y^{1-\lambda}}{M_{r}^{\lambda}(|z|) \Gamma\left(\lambda+\frac{1}{2}\right)}\right]^{\frac{1}{2}} \sum_{n=0}^{\infty} \frac{\left(y \frac{d}{d y}-y+1\right)_{n}}{\prod_{k=1}^{r-1}\left(\lambda+k-\frac{1}{2}\right)_{n}} \frac{(-z)^{n}}{n !} y^{\lambda-\frac{1}{2}}\right|_{y=x^{2}} \\
& =\left.\left[\frac{2 e^{-y} y^{1-\lambda}}{M_{r}^{\lambda}(|z|) \Gamma\left(\lambda+\frac{1}{2}\right)}\right]^{\frac{1}{2}}{ }_{1} F_{r-1}\left(\left[y \frac{d}{d y}-y+1\right],\left[\lambda+\frac{1}{2}, \ldots, \lambda-\frac{3}{2}+r\right],-z\right) y^{\lambda-\frac{1}{2}}\right|_{y=x^{2}}
\end{aligned}
$$

where $(\alpha)_{n}=\frac{\Gamma(\alpha+n)}{\Gamma(\alpha)}$, denotes the Pochhammer symbol. For instance, the explicit compact 
forms of $|z\rangle_{2}^{\lambda}$ and $|z\rangle_{3}^{\lambda}$ are

$$
\begin{aligned}
\langle x \mid z\rangle_{2}^{\lambda} & =\left[\left(-\frac{z}{|z|}\right)^{\frac{1}{2}-\lambda} \frac{2 x}{I_{\lambda-\frac{1}{2}}(2|z|)}\right] e^{-z-\frac{x^{2}}{2}} J_{\lambda-\frac{1}{2}}(2 i x \sqrt{z}), \\
\langle x \mid z\rangle_{3}^{\lambda} & =\frac{1}{\sqrt{{ }_{1} F_{4}\left(\left[\lambda+\frac{1}{2}\right],\left[\lambda+\frac{1}{2}, \lambda+\frac{1}{2}, \lambda+\frac{3}{2}, \lambda+\frac{3}{2}\right],|z|^{2}\right)}} \\
& \times\left.\sqrt{2} e^{-x^{2}} x^{2(1-\lambda)}{ }_{1} F_{2}\left(\left[y \frac{d}{d y}-y+1\right],\left[\lambda+\frac{1}{2}, \lambda+\frac{3}{2}\right],-z\right) y^{\lambda-\frac{1}{2}}\right|_{y=x^{2}},
\end{aligned}
$$

in which $I_{\lambda-\frac{1}{2}}(x)$ is the modified Bessel function of first type and $J_{\lambda-\frac{1}{2}}(x)$ is the ordinary Bessel function defined as $J_{\lambda-\frac{1}{2}}(x)=\sum_{m=0}^{\infty} \frac{(-1)^{m}\left(\frac{x}{2}\right)^{\lambda+2 m-\frac{1}{2}}}{m ! \Gamma\left(\lambda+m+\frac{1}{2}\right)}$ [50].

$\diamond$ Time Evolution of generalized su $(1,1)$ CSs

Due to the relations (2) and (5c), we have

$$
H^{\lambda}|n, \lambda\rangle=\left(2 n+\lambda+\frac{1}{2}\right)|n, \lambda\rangle .
$$

Then the CSs (8) evolve in time as

$$
\begin{aligned}
e^{-i t H^{\lambda}}|z\rangle_{r}^{\lambda} & =\frac{e^{-i t\left(\lambda+\frac{1}{2}\right)}}{\sqrt{M_{r}^{\lambda}(|z|)}} \sum_{n=0}^{\infty}\left(z e^{-i 2 t}\right)^{n} \prod_{k=1}^{r-1} \frac{\Gamma\left(\lambda+k-\frac{1}{2}\right)}{\Gamma\left(n+\lambda+k-\frac{1}{2}\right)} \sqrt{\frac{\Gamma\left(n+\lambda+\frac{1}{2}\right)}{\Gamma\left(\lambda+\frac{1}{2}\right) \Gamma(n+1)}}|n, \lambda\rangle \\
& =e^{-i t\left(\lambda+\frac{1}{2}\right)}\left|z e^{-i 2 t}\right\rangle_{r}^{\lambda},
\end{aligned}
$$

and confirm that $|z\rangle_{r}^{\lambda}$ are temporally stable.

\section{Non-classical properties of $|z\rangle_{r}^{\lambda}$}

In this section, we will set up detailed studies on statistical properties of constructed GNCS. For this reason, proportional nonlinear function associated to them are introduced. Moreover, to analyze their statistical behavior, some of the characters including the second-order correlation function, Mandel's parameter and quadrature squeezing are evaluated. It should be mentioned that squeezing or antibunching (negativity of Mandel parameter) are sufficient (unnecessary) for a state to belong to nonclassical states [53].

\section{$\diamond$ Nonlinearity function}

The question we pose now is whether the $s u(1,1)$ CSs constructed above can be defined as the eigenstates of the non-Hermitian and deformed annihilation operator $f(\hat{N}) J_{-}^{\lambda}$, i.e.

$$
f(\hat{N}) J_{-}^{\lambda}|z\rangle_{r}^{\lambda}=z|z\rangle_{r}^{\lambda}
$$

where $f(\hat{N})$, is determined in terms of the number operator $11 \hat{N}\left(=J_{3}^{\lambda}-\frac{\lambda}{2}-\frac{1}{4}\right)$, plays an important role as a nonlinearity function [25]. Combining definition of the generalized

\footnotetext{
${ }^{1} \hat{N}|n, \lambda\rangle=n|n, \lambda\rangle$.
} 
$s u(1,1)$ CSs (8) and laddering relations (5), we get

$$
\left[\frac{\Gamma\left(\hat{N}+\lambda+r-\frac{1}{2}\right)}{\Gamma\left(\hat{N}+\lambda+\frac{3}{2}\right)}\right] J_{-}^{\lambda}|z\rangle_{r}^{\lambda}=z|z\rangle_{r}^{\lambda} .
$$

So $|z\rangle_{r}^{\lambda}$ can be identified as new classes of $s u(1,1)$ NCSs [54], with characterized nonlinearity functions, $\frac{\Gamma\left(\hat{N}+\lambda+r-\frac{1}{2}\right)}{\Gamma\left(\hat{N}+\lambda+\frac{3}{2}\right)}$. Obviously $f(\hat{N}) \longrightarrow 1$ when $r \longrightarrow 2$.

\section{$\diamond s u(1,1)$ squeezing}

We introduce two generalized Hermitian quadrature operators $X_{1}$ and $X_{2}$

$$
X_{1}^{\lambda}=\frac{J_{+}^{\lambda}+J_{-}^{\lambda}}{2}, \quad X_{2}^{\lambda}=\frac{J_{-}^{\lambda}-J_{+}^{\lambda}}{2 i}
$$

with the commutation relation $\left[X_{1}^{\lambda}, X_{2}^{\lambda}\right]=i J_{3}^{\lambda}$. From this communication relation the uncertainty relation for the variances of the quadrature operators $X_{i}$ follows

$$
\left\langle\left(\Delta X_{1}^{\lambda}\right)^{2}\right\rangle\left\langle\left(\Delta X_{2}^{\lambda}\right)^{2}\right\rangle \geq \frac{\left|\left\langle J_{3}^{\lambda}\right\rangle\right|^{2}}{4}
$$

where $\left\langle\left(\Delta X_{1}^{\lambda}\right)^{2}\right\rangle=\left\langle\left(X_{1}^{\lambda}\right)^{2}\right\rangle-\left\langle X_{1}^{\lambda}\right\rangle^{2}$ and the angular brackets denote averaging over an arbitrary normalizable state for which the mean values are well defined, $\left\langle X_{i}\right\rangle={ }_{r}^{\lambda}\left\langle z\left|X_{i}\right| z\right\rangle_{r}^{\lambda}$. Following Walls (1983) as well as Wodkiewicz and Eberly (1985) [55, 38] we will say that the state is $s u(1,1)$ squeezed if the condition

$$
\left\langle\left(\Delta X_{i}^{\lambda}\right)^{2}\right\rangle<\frac{\left|\left\langle J_{3}^{\lambda}\right\rangle\right|}{2}, \text { for } i=1 \text { or } 2
$$

is fulfilled. In other words, a set of quantum states are called SSs if they have less uncertainty in one quadrature $\left(X_{1}\right.$ or $\left.X_{2}\right)$ than CSs. To measure the degree of the $s u(1,1)$ squeezing we introduce the squeezing factor $S_{i}^{\lambda}[56]$

$$
S_{i}^{\lambda}=\frac{\left\langle\left(\Delta X_{i}^{\lambda}\right)^{2}\right\rangle-\frac{\left|\left\langle J_{3}^{\lambda}\right\rangle\right|}{2}}{\frac{\left|\left\langle J_{3}^{\lambda}\right\rangle\right|}{2}},
$$

it leads that the $s u(1,1)$ squeezing condition takes on the simple form $S_{i}^{\lambda}<0$, however maximally squeezing is obtained for $S_{i}^{\lambda}=-1$. By using of the mean values of the generators of the $s u(1,1)$ Lie algebra, one can derive that uncertainty in the quadrature operators $X_{i}$ can be expressed as the following forms

$$
\left\langle\left(\Delta X_{1(2)}^{\lambda}\right)^{2}\right\rangle=\frac{2\left\langle J_{+}^{\lambda} J_{-}^{\lambda}\right\rangle+2\left\langle J_{3}^{\lambda}\right\rangle \pm\left\langle J_{+}^{\lambda^{2}}+J_{-}^{\lambda^{2}}\right\rangle-\left\langle J_{-}^{\lambda} \pm J_{+}^{\lambda}\right\rangle^{2}}{4} .
$$



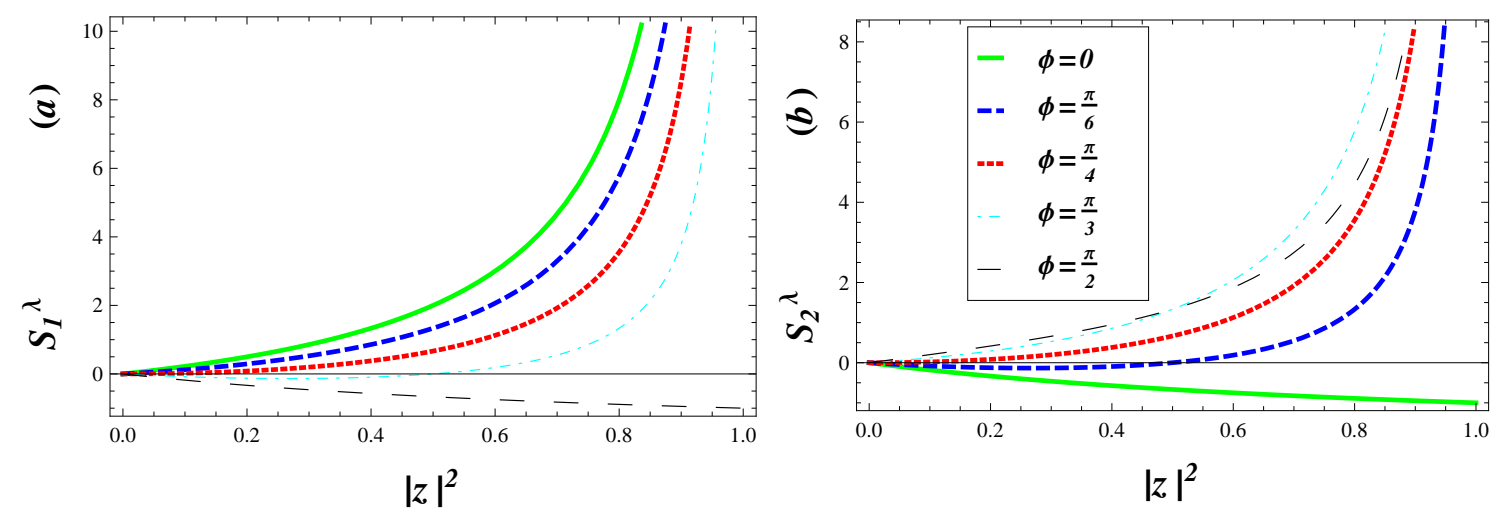

Figure 2:

- Figure. 2. (a) and (b) illustrate squeezing in the $X_{1}^{\lambda}$ and $X_{2}^{\lambda}$ quadratures, respectively, against $|z|^{2}$ for $r=1$ and different values of $\phi$.

where we have the relations

$$
\begin{aligned}
& \left\langle J_{+}^{\lambda}\right\rangle=\overline{\left\langle J_{-}^{\lambda}\right\rangle}=\frac{\Gamma\left(\lambda+\frac{3}{2}\right)}{\Gamma\left(\lambda+r-\frac{1}{2}\right)} \bar{z} \\
& \times \frac{{ }_{2} F_{2 r-1}\left(\left[\lambda+\frac{1}{2}, \lambda+\frac{3}{2}\right],\left[\lambda+\frac{1}{2}, \lambda+\frac{1}{2}, \ldots, \lambda+r-\frac{3}{2}, \lambda+r-\frac{3}{2}, \lambda+r-\frac{1}{2}\right],|z|^{2}\right)}{{ }_{1} F_{2 r-2}\left(\left[\lambda+\frac{1}{2}\right],\left[\lambda+\frac{1}{2}, \lambda+\frac{1}{2}, \ldots, \lambda+r-\frac{3}{2}, \lambda+r-\frac{3}{2}\right],|z|^{2}\right)} \\
& \left\langle J_{+}^{\lambda^{2}}\right\rangle=\overline{\left\langle J_{-}^{\lambda^{2}}\right\rangle}=\frac{\Gamma\left(\lambda+\frac{3}{2}\right) \Gamma\left(\lambda+\frac{5}{2}\right)}{\Gamma\left(\lambda+r-\frac{1}{2}\right) \Gamma\left(\lambda+r+\frac{1}{2}\right)} \bar{z}^{2} \\
& \times \frac{{ }_{1} F_{2 r-2}\left(\left[\lambda+\frac{5}{2}\right],\left[\lambda+\frac{1}{2}, \lambda+\frac{3}{2}, \ldots, \lambda+r-\frac{1}{2}, \lambda+r+\frac{1}{2}\right],|z|^{2}\right)}{{ }_{1} F_{2 r-2}\left(\left[\lambda+\frac{1}{2}\right],\left[\lambda+\frac{1}{2}, \lambda+\frac{1}{2}, \ldots, \lambda+r-\frac{3}{2}, \lambda+r-\frac{3}{2}\right],|z|^{2}\right)}, \\
& \left\langle J_{+}^{\lambda} J_{-}^{\lambda}\right\rangle=\frac{\Gamma\left(\lambda+\frac{3}{2}\right)^{2}}{\Gamma\left(\lambda+r-\frac{1}{2}\right)^{2}}|z|^{2} \\
& \times \frac{{ }_{3} F_{2 r}\left(\left[\lambda+\frac{1}{2}, \lambda+\frac{3}{2}, \lambda+\frac{3}{2}\right],\left[\lambda+\frac{1}{2}, \lambda+\frac{1}{2} \ldots, \lambda+r-\frac{1}{2}, \lambda+r-\frac{1}{2}\right],|z|^{2}\right)}{{ }_{1} F_{2 r-2}\left(\left[\lambda+\frac{1}{2}\right],\left[\lambda+\frac{1}{2}, \lambda+\frac{1}{2}, \ldots, \lambda+r-\frac{3}{2}, \lambda+r-\frac{3}{2}\right],|z|^{2}\right)}, \\
& \left\langle J_{3}^{\lambda}\right\rangle=\left(\lambda+\frac{1}{2}\right) \\
& \times \frac{{ }_{2} F_{2 r-1}\left(\left[\frac{\lambda}{2}+\frac{5}{4}, \lambda+\frac{1}{2}\right],\left[\frac{\lambda}{2}+\frac{1}{4}, \lambda+\frac{1}{2}, \lambda+\frac{3}{2}, \lambda+\frac{3}{2}, \ldots, \lambda+r-\frac{3}{2}, \lambda+r-\frac{3}{2}\right],|z|^{2}\right)}{{ }_{1} F_{2 r-2}\left(\left[\lambda+\frac{1}{2}\right],\left[\lambda+\frac{1}{2}, \lambda+\frac{1}{2}, \ldots, \lambda+r-\frac{3}{2}, \lambda+r-\frac{3}{2}\right],|z|^{2}\right)} .
\end{aligned}
$$

They result that, $\left\langle\left(\Delta X_{1(2)}^{\lambda}\right)^{2}\right\rangle$ as well as $S_{1(2)}^{\lambda}$, for any value of $\lambda$, are efficiently dependent 
on the complex variable $z\left(=|z| e^{i \varphi}\right)$ and the deformation parameter $r$.

Case $r=1$ :

Our calculations show that the squeezing factor $S_{1}^{\lambda}$ is really independent of $\lambda$. It illustrates that squeezing properties in the $X_{1}$ quadrature arise when $\varphi$ is increased and culminates where $\varphi$ reaches $\frac{\pi}{2}$ as well as $|z| \rightarrow 1$ (see figure 2(a)). However, figure 2(b) implies that squeezing properties in the $X_{2}$ quadrature is considerable where $\varphi$ is decreased. It becomes maximal, $S_{2}^{\lambda} \rightarrow-1$, if $\varphi$ tends to zero [56, 46].

口 Case $r=2$ :

Clearly, for the case $r=2$ we would not expect to take squeezing neither in $X_{1}$ nor in $X_{2}$ quadratures [56, 46].

- Case $r \geq 3$ :

In Figures 3 we plot the squeezing parameter $S_{1}^{\lambda}$ and $S_{2}^{\lambda}$ as a function of $|z|^{2}$ for different values of $r(=3,4$ and 5$)$ as well as $\lambda\left(=-\frac{1}{4}, \frac{1}{4}, 1\right)$, here we choose the phase $\varphi=0$. According to Figures $3(a, c, e)$, it is visible, the squeezing parameter $S_{1}^{\lambda}$ is less than zero for the all regions of $|z|^{2}$. This indicate that for $\varphi=0$, the quadrature squeezing occur only in the $X_{1}$ component for the all regions of $|z|^{2}$. Also, we show in Figures 4 the squeezing parameter $S_{1}^{\lambda}$ and $S_{2}^{\lambda}$ for different values of $\varphi\left(=0, \frac{\pi}{6}, \frac{\pi}{4}, \frac{\pi}{3}\right.$, and $\left.\frac{\pi}{2}\right)$ for fixed $r=4$. From Figures $3(a, c, e)$, and Figures $3(b, d, f)$ we find that while quadrature squeezing in the $X_{1}$ component occur for $\phi=0$ and $\phi=\frac{\pi}{6}$, there is quadrature squeezing effect in the $X_{2}$ component for $\phi=\frac{\pi}{3}$ and $\phi=\frac{\pi}{2}$.

\section{$\diamond$ Anti-bunching effect and sub-Poissonian statistics}

Now we are in a position to study the anti-bunching effect as well as the statistics of $|z\rangle_{r}^{\lambda}$ given by equation (8). We introduce the second-order correlation function for these states

$$
\left(g^{(2)}\right)_{r}^{\lambda}\left(|z|^{2}\right)=\frac{\left\langle\hat{N}^{2}\right\rangle_{r}^{\lambda}-\langle\hat{N}\rangle_{r}^{\lambda}}{\langle\hat{N}\rangle_{r}^{\lambda^{2}}},
$$

furthermore, the inherent statistical properties of the $|z\rangle_{r}^{\lambda}$ follows also from calculating the Mandel parameter $Q_{r}^{\lambda}\left(|z|^{2}\right)^{2}$

$$
Q_{r}^{\lambda}\left(|z|^{2}\right)=\langle\hat{N}\rangle_{r}^{\lambda}\left[\left(g^{(2)}\right)_{r}^{\lambda}\left(|z|^{2}\right)-1\right]
$$

In order to find the function $\left(g^{(2)}\right)_{r}^{\lambda}\left(|z|^{2}\right)$, also Mandel parameter $Q_{r}^{\lambda}\left(|z|^{2}\right)$, let us begin with the expectation values of the number operator $\hat{N}$ and of the square of the number operator

\footnotetext{
${ }^{2}$ A state for which $Q_{r}^{\lambda}\left(|z|^{2}\right)>0\left(\right.$ or $\left.\left(g^{(2)}\right)_{r}^{\lambda}\left(|z|^{2}\right)>1\right)$ is called super-Poissonian (bunching effect), if $Q=0$ (or $\left.g^{(2)}=1\right)$ the state is called Poissonian, while a state for which $Q<0$ (or $\left.g^{(2)}<1\right)$ is, also, called sub-Poissonian (antibunching effect).
} 

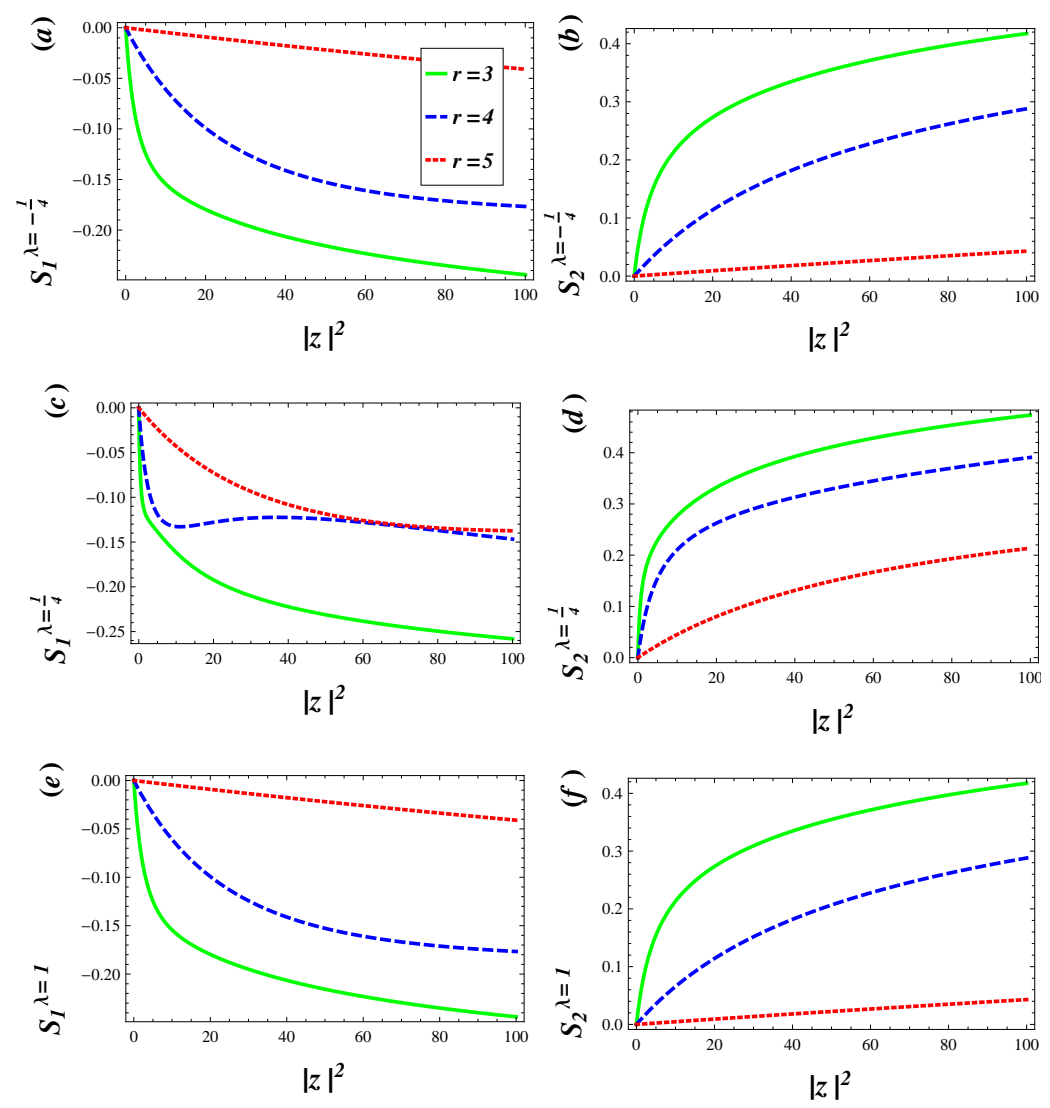

Figure 3:

- Figure. 3. Graphs of uncertainty in the field quadratures $X_{1}^{\lambda}(a, c, e)$ and $X_{2}^{\lambda}(b, d, f)$, respectively versus $|z|^{2}$ for different values of $r$ as well as different $\lambda$ while we choose the phase $\varphi=0$. 

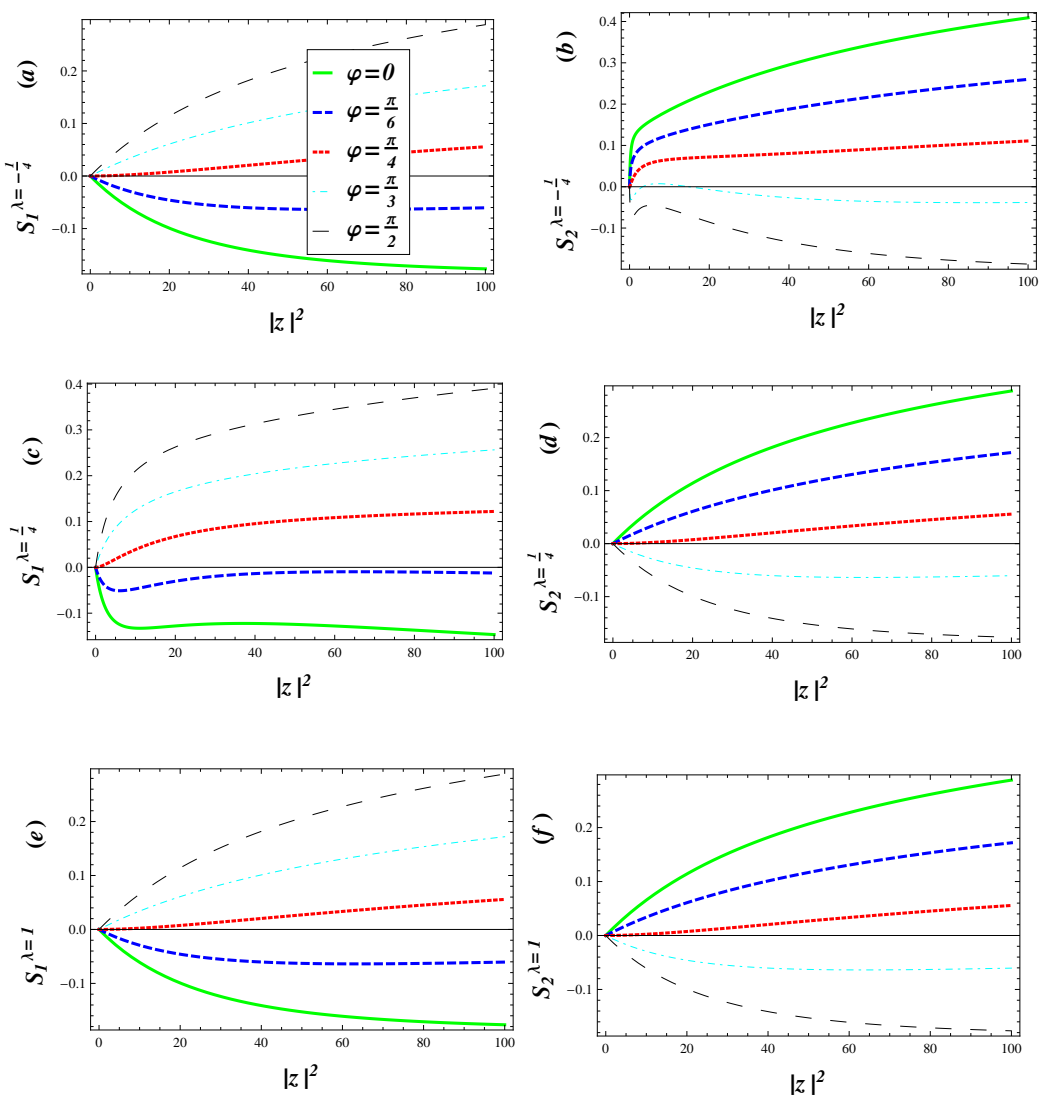

Figure 4:

- Figure. 4. Squeezing in the $X_{1}^{\lambda}$ and $X_{2}^{\lambda}$ quadratures have been shown in figures $(a, c, e)$ and $(b, d, f)$ respectively, against for $r=4$ and different values of $\varphi$. 


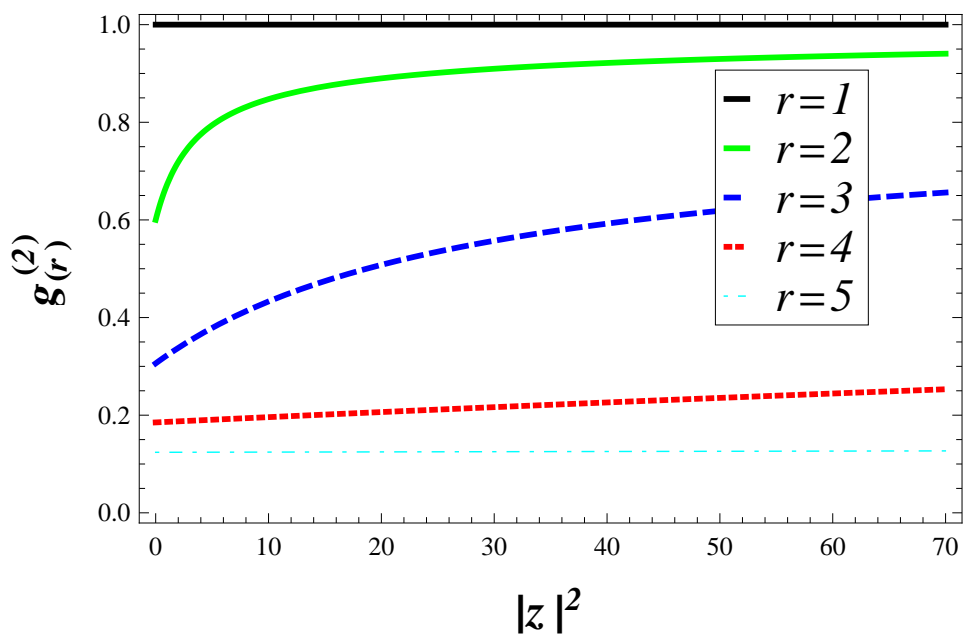

Figure 5:

- Figure. 5. Plot of the correlation function $\left(g^{(2)}\right)_{r}^{\lambda=0}\left(|z|^{2}\right)$ versus $|z|^{2}$ for different values of $r$. The solid curve, $\left(g^{(2)}\right)_{r}=1$ corresponds to the canonical CSs.

$\hat{N}^{2}$ in the basis of the Fock space states $|n, \lambda\rangle$

$$
\begin{gathered}
\langle\hat{N}\rangle_{r}^{\lambda}=\frac{|z|^{2}}{\lambda+\frac{1}{2}}\left(\frac{\Gamma\left(\lambda+\frac{5}{2}\right)}{\Gamma\left(\lambda+r+\frac{1}{2}\right)}\right)^{2} \\
\times \frac{{ }_{1} F_{2 r-2}\left(\left[\lambda+\frac{3}{2}\right],\left[\lambda+\frac{3}{2}, \lambda+\frac{3}{2}, \ldots, \lambda+r-\frac{1}{2}, \lambda+r-\frac{1}{2}\right],|z|^{2}\right)}{{ }_{1} F_{2 r-2}\left(\left[\lambda+\frac{1}{2}\right],\left[\lambda+\frac{1}{2}, \lambda+\frac{1}{2}, \ldots, \lambda+r-\frac{3}{2}, \lambda+r-\frac{3}{2}\right],|z|^{2}\right)}, \\
\left\langle\hat{N}^{2}\right\rangle_{r}^{\lambda}=\frac{|z|^{2}}{\lambda+\frac{1}{2}}\left(\frac{\Gamma\left(\lambda+\frac{3}{2}\right)}{\Gamma\left(\lambda+r-\frac{1}{2}\right)}\right)^{2} \\
\quad \times \frac{{ }_{2} F_{2 r-1}\left(\left[2, \lambda+\frac{3}{2}\right],\left[1, \lambda+\frac{3}{2}, \lambda+\frac{3}{2}, \ldots, \lambda+r-\frac{1}{2}, \lambda+r-\frac{1}{2}\right],|z|^{2}\right)}{{ }_{1} F_{2 r-2}\left(\left[\lambda+\frac{1}{2}\right],\left[\lambda+\frac{1}{2}, \lambda+\frac{1}{2}, \ldots, \lambda+r-\frac{3}{2}, \lambda+r-\frac{3}{2}\right],|z|^{2}\right)} .
\end{gathered}
$$

For the case $r=1$, the second-order correlation function can be calculated to be taken as $\left(g^{(2)}\right)_{r=1}^{\lambda}\left(|z|^{2}\right)=1+\frac{1}{1+\frac{\lambda}{2}}>1$. This guaranties that $|z\rangle_{1}^{\lambda}$ exhibits a fully bunching effect, or super-Poissonian statistics [56. But this situation is changed when $r$ and $\lambda$ are enhanced. In Figure $5,\left(g^{(2)}\right)_{r}^{\lambda}\left(|z|^{2}\right)$ has been plotted in terms of $|z|^{2}$ for several values of $r(=2,3,4$ and $5)$. From this Figure, it is observed that this parameter is less than one, for all regions and so the statistics of the $|z\rangle_{r}^{\lambda}$ is fully sub-Poissonian. Recalling that each of the nonclassicality indicators is sufficient (not necessary) for a state to be nonclassical, we observed that the generalized $s u(1,1)$ CSs are indeed nonclassical states.

\section{Conclusions}

Based on a new approach, broad range of generalized su(1,1) CSs for PHO are constructed. These states realize a resolution of the identity with positive measures on the complex plane. 
Non-classical properties of such states have been reviewed in detail. It has been shown that they have squeezing properties and follow the sub-Poissonian statistics. For these reasons the constructed generalized $s u(1,1)$ CSs can be termed as nonclassical states. Generally, the approach presented here provides a unified method to construct all relavant CSs introduced in different ways (the Klauder-Perelomov and Barut-Girardello CSs). The advantage of this approach is that, one need only the raising operators associated with the systems under consideration without addressing the dynamical symmetry of system. Also, this approach

can be used to construct new type CSs for exactly solvable models in the framework of the quantum mechanics in which the laddering operators are dependent to the quantum modes, such as the Hydrogen atom and the Morse model.

\section{References}

[1] Schrodinger E 1926 Ann. Phys., Lpz 79361.

[2] Glauber R J 1963 Phys. Rev 1302529.

[3] Glauber R J 1963 Phys. Rev 1312766.

[4] Klauder J R 1963 J. Math. Phys 41055.

[5] Klauder J R 1963 J. Math. Phys 41058.

[6] Sudarshan E C G 1963 Phys. Rev. Lett 10277.

[7] Barut A O and Girardello L 1971 Commun. Math. Phys 2141.

[8] Perelomov A M 1972 Commun. Math. Phys 26222.

[9] Gazeau J P and Klauder J R 1999 J. Phys. A: Math. Gen 32123.

[10] Antoine J P, Gazeau J P, Monceau P, Klauder J R and Penson K A 2001 J. Math. Phys 422349.

[11] Fukui T and Aizawa N 1993 Phys. Lett. A 180308.

[12] Chenaghlou A and Fakhri H 2002 Mod. Phys. Lett. A 171701.

[13] Stoler D 1970 Phys. Rev. D 13217.

[14] Kimble H J, Dagenais M and Mandel L 1977 Phys. Rev. Lett 39691.

[15] Short R and Mandel L 1983 Phys. Rev. Lett 51384.

[16] Teich M C and Saleh B E A 1985 J. Opt. Soc. Am. B 2275.

[17] Slusher R E, Hollberg L W, Yurke B, Mertz J C and Valley J F 1985 Phys. Rev. Lett 552409.

[18] Wu L -A , Kimble H J, Hall J L and Wu, H 1986 Phys. Rev. Lett 572520. 
[19] Biedenharn L C 1989 J. Phys. A: Math. Gen 22 L873.

[20] Yurke B and Stoler D 1986 Phys. Rev. Lett 5713.

[21] Noel M W and Stroud C R 1996 Jr. in Coherence and Quantum Optics VII Plenum, New York 563564.

[22] de Matos Filho R L and Vogel W 1996 Phys. Rev. Lett 76608.

[23] Monroe C, Meekhof D M, King B E and Wineland D J 1996 Science 2721131.

[24] Manko V I, Marmo G, Sudarshan E C G, Zaccaria F 1997 Phys. Scripta 55528.

[25] de Matos Filho R L and Vogel W 1996 Phys. Rev. A 544560.

[26] Raffa F. A, Rasetti M and Genovese M 2012 Phys. Lett. A 376330.

[27] Kis Z, Vogel W and Davidovich L 2001 Phys. Rev. A 64033401.

[28] Agarwal G S and Tara K 1991 Phys. Rev. A 43492.

[29] Walls D F 1983 Nature 306141.

[30] Wang X G 2001 Can. J. Phys 79(5) 833.

[31] Honarasa G R, Tavassoly M K, Hatamia M, Roknizadeh R, 2011 Physica. A 3901381.

[32] Piroozi E and Tavassoly M K, 2012 J. Phys. A: Math. Theor. 45135301.

[33] Roknizadeh R and Tavassoly M K, 2004 J. Phys. A: Math. Gen. 378111.

[34] Roknizadeh R and Tavassoly M K, 2005 J. Math. Phys. 46042110.

[35] Perelemov A M 1986 Generalized Coherent States and Their Applications Springer, Berlin.

[36] Vourdas A 1990 Phys. Rev. A 411653.

[37] Zhang W -M, Feng D H and Gilmore R 1990 Rev. Mod. Phys 62867.

[38] Wodkiewwicz K and Eberly J H 1985 J. opt . Soc. Am. B 2 458K.

[39] Obada A-S F and Al-Kader G M Abd, J. Opt. B: Quantum Semiclass. Opt. 72005 S635S642; Obada A-S F and Al-Kader G M Abd 2007 Eur. Phys. J. D 41, 189.

[40] Goldman I I and Krivchenkov V D 1961 Problems in Quantum Mechanics (Pergamon, Oxford).

[41] Hall R L and Saad N (2000) J. Phys. A: Math. Gen. 33 569-578.

[42] Hall R L, Saad N and von Keviczky A 2002 J. Math. Phys. 4394.

[43] Saad N, Hall R L and von Keviczky A 2003 J. Phys. A: Math. Gen. 36487. 
[44] Agarwal G. S and Chaturvedi S J. Phys. A 1995285747.

[45] Fakhri H, Dehghani A and Mojaveri B 2009 Int. J. Quantum Chem. 1091228.

[46] Tavassoly M K and Jalali H R, arxiv:303.4105v1 [quant-ph] (2013).

[47] Dehghani A and Mojaveri B 2012 J. Phys. A: Math. Theor 45095304.

[48] Fu H C and Sasaki R 1996 Phys. Rev. A. 533836.

[49] Dodonov V V, Malkin I A and Man'ko V I 1974 Physica 72597.

[50] Gradshteyn I S and Ryzhik I M 2000 Table of Integrals, Series, and Products (San Diego, CA: Academic).

[51] Appl T and Schiller D H 2004, J. Phys. A: Math. Gen 372731.

[52] Cessa H M , New expression for Laguerre and Hermite polynomials arXiv:0809.2259v1 [math-ph] (2008).

[53] Shchukin E V and Vogel W, Phys. Rev. A, 72 (2005) 043808.

[54] Wang X G, Int. J. Mod. Phys. B. 14(10)(2000)1093-1104.

[55] Walls D F 1983 Nature 306141.

[56] Buzek V 1990 J. Mod. Opt 37303. 